\title{
CAUSAS DE RETRABALHO DE PRODUTOS PARA SAÚDE NO CENTRO DE MATERIAIS E ESTERILIZAÇÃO
}

Causes of rework of health products at the material and sterilization center

Causas de retrabajo de productos para salud en el centro de materiales y esterilización

André Luiz Silva Alvim* ${ }^{1 *}$ Karinne Ferreira de Souza²

RESUMO: Objetivo: Identificar as principais causas de retrabalho de produtos para saúde (PPS) detectadas no Centro de Materiais e Esterilização (CME) de um hospital particular de Belo Horizonte, Minas Gerais, Brasil. Método: Estudo descritivo, desenvolvido em um CME de classe II de um hospital privado de Belo Horizonte. Foi realizada análise documental entre janeiro e junho de 2016, por meio de 181 registros de checklist e documentos de trabalho. Para tratamento dos dados, utilizou-se análise estatística descritiva para apresentação de valores absolutos e porcentagens geradas pelo programa Epilnfo $7^{\circledR}$. Resultados: Foram encontrados 605 itens de retrabalho, que representaram uma taxa de $0,75 \%$ do total de caixas e pacotes produzidos. As principais causas foram relacionadas a produtos vencidos (74\%) e resíduos orgânicos pós-esterilização (13\%). Conclusão: O enfermeiro deve trabalhar com a equipe para redução das causas de retrabalho que impactam em desperdícios e custos desnecessários.

Palavras-chave: Esterilização. Indicadores. Instrumentos cirúrgicos. Enfermagem.

ABSTRACT: Objective: To identify the main causes of rework of health products detected at the Materials and Sterilization Center of a private hospital in Belo Horizonte, Minas Gerais, Brazil. Methods: A descriptive study was developed in a class II Sterilization Center of a private hospital in Belo Horizonte, Minas Gerais, Brazil. Documentary analysis was performed between January and June 2016, through 181 checklist records and working documents. For data treatment, descriptive statistical analysis was used for the presentation of absolute values and percentages generated by the Epi Info $7^{\circledR}$ program. Results: We found 605 rework items, which represented the rate of $0.75 \%$ of the total boxes and packages produced. The main causes were related to overdue products $(74 \%)$ and post-sterilization organic wastes $(13 \%)$. Conclusion: The nurse must work with its team to reduce the causes of rework, that impact unnecessary waste and costs.

Keywords: Sterilization. Indicators. Surgical Instruments. Nursing.

RESUMEN: Objetivo: Identificar las principales causas de retrabajo de productos para salud (PPS) detectadas en el Centro de Materiales y Esterilización (CME) de un hospital privado de Belo Horizonte, Minas Gerais, Brasil. Método: Estudio descriptivo, desarrollado en un CME de clase II de un hospital privado de Belo Horizonte. Se realizó un análisis documental entre enero y junio de 2016, a través de 181 registros de checklist y documentos de trabajo. Para el tratamiento de los datos, se utilizó análisis estadístico descriptivo para presentación de valores absolutos y porcentajes generados por el programa EpiInfo $7^{\circledR}$. Resultados: Se encontraron 605 ítems de retrabajo, que representaron una tasa del $0,75 \%$ del total de cajas y paquetes producidos. Las principales causas fueron relacionadas con productos vencidos (74\%) y residuos orgánicos post-esterilización (13\%). Conclusión: El enfermero debe trabajar con un equipo para reducir las causas de retrabajo, que impactan en desperdicios y costos innecesarios.

Palabras clave: Esterilización. Indicadores. Instrumentos Quirúrgicos. Enfermería.

'Enfermeiro mestre pela Unidade Federal de Minas Gerais; MBA em Auditoria e Gestão da Qualidade aplicada a Serviços de Saúde - Belo Horizonte (MG), Brasil.

2Enfermeira mestre em Administração pela Faculdade de Estudos Administrativos de Minas Gerais; pós-graduada em Gestão Estratégica e Infecção Hospitalar - Belo Horizonte (MG), Brasil.

*Autor correspondente: andrealvim1@hotmail.com

Recebido: 19/06/2017 - Aprovado: 16/11/2017

DOl: $10.5327 / Z 1414-4425201800010002$ 


\section{INTRODUÇÃO}

O Centro de Materiais e Esterilização (CME) é destinado ao processamento de produtos para saúde (PPS) envolvidos nos procedimentos críticos e semicríticos do paciente. Essa unidade funcional possui história que vem acompanhando os procedimentos cirúrgicos, a fim de zelar pela prevenção de Infecções Relacionadas à Assistência à Saúde (IRAS) ${ }^{1,2}$.

Nesse sentido, a busca pela qualidade no processamento em PPS vem sendo considerada um requisito essencial para o CME. Entre as diversas ferramentas disponíveis para o monitoramento dos resultados referentes aos processos de trabalho, destacam-se os indicadores de qualidade ${ }^{3-5}$.

Os indicadores são medidas quantitativas que refletem a realidade das mudanças ocorridas em uma determinada realidade e permitem o direcionamento de condutas para elevação do desempenho organizacional pelos gestores. O CME conta com vários indicadores que podem ser incorporados nos diversos processos; no entanto, não foram encontrados estudos na literatura que avaliassem e quantificassem causas de retrabalho dos PPS ${ }^{3-5}$.

Essa lacuna do conhecimento motivou os pesquisadores a desvelarem a seguinte questão norteadora: Quais são as principais causas de retrabalho detectadas no CME? Este estudo poderá contribuir para o conhecimento de fatores que interferem nos custos do CME e que podem desequilibrar recursos financeiros destinados à unidade de apoio, considerando a atual situação econômica do país ${ }^{6}$.

\section{OBJETIVO}

Identificar as principais causas de retrabalho dos produtos para saúde, detectadas no CME.

\section{MÉTODO}

Trata-se de um estudo descritivo, de natureza quantitativa, desenvolvido em um CME de classe II de um hospital geral particular de Belo Horizonte, Minas Gerais, Brasil. O CME classe II é definido como aquele que realiza o processamento de PPS não críticos, semicríticos e críticos, de conformação complexa e não complexa, passíveis de processamento ${ }^{1}$.

Em 2010, entre os diversos indicadores atrelados ao CME do hospital de estudo, iniciou-se avaliação da taxa de retrabalho dos PPS. Desde então, esse indicador vem contribuindo para a verificação dos fatores que impactam nas causas de desperdícios e custos desnecessários. No hospital de estudo, o prazo de validade dos invólucros/barreiras de proteção em tecido não tecido (Spunbond Meltblown Spunbond (SMS)) e grau cirúrgico contempla, respectivamente, 30 e 180 dias. Na esterilização à baixa temperatura, o Tyvec ${ }^{\circledR}$ vence após 365 dias.

Foi realizada análise documental entre janeiro e junho de 2016 pelo primeiro pesquisador, por meio de 181 registros de checklist e documentos de trabalho que se encontravam disponíveis em forma física e lançados no sistema de gestão da qualidade do hospital. As causas de retrabalho foram registradas pelo técnico de enfermagem alocado no arsenal em checklist específico, contendo as seguintes variáveis: produtos vencidos, resíduo orgânico pós-esterilização, perda ou rasura da etiqueta, etiqueta incorreta, pacotes molhados, embalagem não íntegra e resíduo de oxidação.

Após captação dos dados, utilizou-se uma planilha para agrupamento dos dados registrados. Ao final, foram encontrados 605 itens de retrabalho no período proposto para o estudo. Os dados foram lançados no programa Microsoft Excel ${ }^{\circledR}$. Para tratamento dos dados foi utilizada análise estatística descritiva para apresentação de valores absolutos e porcentagens gerados pelo programa Epi Info $7^{\circledR}$. Por se tratar de um estudo que não envolve pesquisa com seres humanos, não foi necessário um parecer do Comitê de Ética em Pesquisa (CEP); no entanto, foi concedida autorização institucional para realização do estudo, elaborada pela gerente de enfermagem.

\section{RESULTADOS}

Foram detectados 605 itens refeitos no total de 80.568 PPS produzidos, representando uma taxa de retrabalho de $0,75 \%$. A Tabela 1 mostra que as principais causas de retrabalho

Tabela 1. Causas de retrabalho dos produtos para saúde detectadas no Centro de Materiais e Esterilização, Belo Horizonte, Minas Gerais, 2016. \begin{tabular}{|l|l|l}
\hline Descrição dos itens de retrabalho & Número & $\%$
\end{tabular}

\begin{tabular}{|l|c|c|}
\hline PPS vencidos & 449 & 74,2 \\
\hline Resíduo orgânico pós-esterilização & 79 & 13,0 \\
\hline Etiqueta incorreta & 18 & 3,0 \\
\hline Pacotes molhados & 18 & 3,0 \\
\hline Perda ou rasura da etiqueta & 17 & 2,8 \\
\hline Embalagem não íntegra & 12 & 2,0 \\
\hline Resíduo de oxidação & 12 & 2,0 \\
\hline Total & 605 & 100,0 \\
\hline
\end{tabular}

PPS: produtos para saúde. 
estavam atreladas aos produtos vencidos $(74,2 \%)$ e à existência de resíduo orgânico pós-esterilização $(13,0 \%)$.

Outras causas de retrabalho obtiveram baixa frequência, como: pacotes molhados $(3,0 \%)$, etiqueta incorreta $(3,0 \%)$, perda ou rasura da etiqueta $(2,8 \%)$, resíduo de oxidação $(2,0 \%)$ e embalagem não íntegra $(2,0 \%)$.

\section{DISCUSSÃO}

O CME é considerado unidade vital de um hospital, que realiza atividades complexas de limpeza, preparo, esterilização e distribuição de PPS para diversas unidades assistenciais. Essas unidades contemplam ações de terceiros, médicos e profissionais de enfermagem realizadas com o paciente ${ }^{1,6-8}$.

Dessa forma, torna-se intrínseco ao setor buscar a qualidade de cada etapa do processamento de PPS a fim de reduzir eventos indesejados. A partir dessa premissa, a incorporação de indicadores de qualidade tornou-se essencial para o monitoramento de resultados de processos de trabalho9.

No período do estudo, verificou-se que os itens de retrabalho por produtos vencidos foram prevalentes quando comparados às outras causas. Esse fato foi atribuído, principalmente, ao prazo de validade expirado e ao consequente não uso pelas áreas assistenciais e cirúrgicas.

Não há um consenso sobre determinação de prazo de validade fixo para os PPS. Autores consideram que caixas cirúrgicas mantiveram-se estéreis mesmo após contaminação proposital por microrganismos e armazenamento inadequado $^{10}$. No entanto, pesquisadores afirmam que esse prazo deve ser estabelecido, levando-se em conta vários fatores, como limpeza, invólucro e armazenamento, que possam garantir a manutenção da esterilidade ${ }^{11,12}$.

Em relação aos resíduos orgânicos pós-esterilização, destaca-se que a limpeza inadequada foi a principal causa atrelada ao retrabalho. No hospital de estudo foram registrados problemas em relação à manutenção das lavadoras ultrassônicas, impedindo que a limpeza automatizada fosse efetiva nessa etapa do processamento.

A limpeza é definida como remoção de sujidade orgânica e inorgânica, redução da carga microbiana presente nos PPS, utilizando água, detergentes, produtos e acessórios de limpeza, por meio de ação mecânica (manual ou automatizada), atuando em superfícies internas (lúmens) e externas, a fim de tornar o produto seguro para o manuseio e preparado para desinfecção ou esterilização ${ }^{1}$. Quando os profissionais do CME não se conscientizam em relação à importância desta etapa e a executam de maneira condescendente ou superficial, a sujidade não será totalmente removida e poderá criar barreiras que protejam os microorganismo ${ }^{2,7,13-15}$.

No CME, a limpeza dos PPS é executada pela equipe de enfermagem. Essa etapa é considerada primordial para garantia da eficácia das etapas que a procedem, evitando que materiais ou instrumentais utilizados no paciente se tornem veículos de contaminação de microrganismos ${ }^{1,2,7}$.

As outras causas de retrabalho obtiveram baixa frequência nos resultados do CME estudado. No entanto, é importante trabalhá-las com toda equipe para promoção de uma gestão de cuidados, a fim de eliminar gastos desnecessários com PPS. Estudos demonstraram que fatores relacionados à qualidade da embalagem utilizada para esterilização, bem como o tipo de material padronizado pelo gestor, podem contribuir para a redução da taxa de retrabalho ${ }^{16,17}$.

O conhecimento das causas de retrabalho para o gerenciamento de custos é importante para diminuição de desperdícios. Autores afirmam que o retrabalho pode estar relacionado com processos institucionais mal estruturados ${ }^{16}$. Assim, a educação continuada dos profissionais, por meio de treinamentos e cursos de reciclagem, é um fator contribuinte para o declínio desse indicador. Além disso, o levantamento das causas de retrabalho pelo enfermeiro do CME com a equipe de enfermagem é essencial para a investigação de falhas de processos e, concomitantemente, elaboração de uma análise de causa-raiz efetiva ${ }^{6,18,19}$.

\section{CONSIDERAÇÕES FINAIS}

É essencial que o enfermeiro atuante no CME trabalhe junto com a equipe de enfermagem para redução das causas de retrabalho relacionadas aos produtos vencidos e resíduos orgânicos pós-esterilização. Com isso, pode-se criar estratégias para gerenciamento de custos, bem como conhecer os fatores que impactam no aumento de gastos relacionados à unidade funcional.

Este indicador permitiu conhecer os fatores de desperdício que impactam na geração de custos desnecessários nos processos do CME. Embora a taxa de retrabalho representasse apenas $0,75 \%$ de todos os PPS produzidos na unidade estudada, é importante a avaliação periódica dos dados para subsidiar a gestão de custos realizada pelo enfermeiro. 


\section{REFERÊNCIAS}

1. Brasil. Ministério da Saúde. Agência Nacional de Vigilância Sanitária. Resolução n. 15, de 15 de março de 2012. Dispõe sobre requisitos de boas práticas para o processamento de produtos para saúde e dá outras providências. Brasília: Diário Oficial da União; 2012.

2. Ouriques $C M$, Machado ME. Enfermagem no processo de esterilização de materiais. Texto Contexto Enferm. 2013;22(3):695-703.

3. Tronchin DM, Melleiro MM, Kurcgant P, Garcia AN, Garzin AC. Subsídios teóricos para a construção e implantação de indicadores de qualidade em saúde. Rev Gaúcha Enferm [Internet]. 2009 [acesso em: 2017 Jun. 20];30(3):542-6. Disponível em: http://seer.ufrgs.br/ RevistaGauchadeEnfermagem/article/view/10412

4. Graziano KU, Lacerda RA; Turrini RT; Bruna CQ; Silva CP; Schmitt C, et al. Indicadores de avaliação do processamento de artigos odontomédico-hospitalares: elaboração e validação. Rev Esc Enferm USP. 2009;43(spe2):1174-80. DOI: 10.1590/S0080-62342009000600005

5. Passos IP, Padoveze MC, Roseira CE, Figueiredo RM. Adaptation and validation of indicators concerning the sterilization process of supplies in Primary Health Care services. Rev Latino-Am Enfermagem. 2015;23(1):148-54. DOI: 10.1590/0104-1169.3518.2536

6. Castilho V, Castro LC, Couto AT, Maia FO, Sasaki NY, Nomura FH, et al. Levantamento das principais fontes de desperdício de unidades assistenciais de um hospital universitário. Rev Esc Enferm USP. 2011;45(Spe):1613-20. DOI:10.1590/S0080-62342011000700012

7. Rutala WA, Weber DJ. Disinfection and sterilization in health care facilities: an overview and current issues. Infect Dis Clin North Am. 2016;30(3):609-37. DOI: 10.1016/j.idc.2016.04.002

8. Luckwü AD, Silva EL, Araújo EC. Exposure factors of the health professional to substances chemicals used in the process of cleaning and disinfecting at the purge sector. Journ Nursing UFPE [Internet]. 2010 [acessoem:2017 Jun. 20];(4)1. Disponível em: http://www.revista. ufpe.br/revistaenfermagem/index.php/revista/article/viewArticle/750

9. Fusco SF, SpiriWC. Analysis of quality indicators of central sterile supply departments at accredited public hospitals. Texto Contextoenferm. 2014;23(2):426-33. DOI: 10.1590/0104-07072014001570013
10. Moriya GA, Graziano KU. Avaliação da manutenção da esterilidade de materiais úmidos/molhados após a esterilização por vapor e armazenamento por 30 dias. Rev Latino-Am Enfermagem [Internet]. 2010 [acesso em: 2017 Jun. 19];18(4):1-7. Disponivel em: http:// www.scielo.br/pdf/rlae/v18n4/pt_18.pdf

11. Neves ZC, Melo DS, Souza AC, Tipple AF, Rodriguez MA. Artigos esterilizados em calor úmido: validação do sistema de guarda. Rev Bras Enferm.2004;57(2):152-6. DOI:10.1590/S0034-71672004000200004

12. Lopes CL, Graziano KU, Pinto TJ. Evaluation of single-use reprocessed laparoscopic instrument sterilization. Rev Latino-AmEnfermagem. 2011;19(2):370-77. DOI:10.1590/S0104-11692011000200020

13. Souza MC, Ceribelli MI. Enfermagem no centro de material esterilizado: a prática da educação continuada. ver Latino-AmEnfermagem. 2004;12(5):767-74. DOI:10.1590/S0104-11692004000500010

14. UchikawaK, Silva A, Psaltikidis EM. Enfermagem em centro de material e esterilização. Barueri: Manole; 2011.

15. William AR, David JW. Guideline for disinfection and sterilization in healthcare facilities. Atlanta; 2008:1-161.

16. Silva PC, SeverianoFilho C. Ocorrência de custos ocultos em operações de serviços: insights sobre observação em uma sociedade de economia mista no Brasil. Gest Prod. 2011;18(3):499-508. DOI: 10.1590/S0104-530X2011000300005

17. Serratine AC, Gonçalves CS, Lucolli IC.Influência do armazenamento e da embalagem na manutenção da esterilidadedo instrumental odontológico. Rev Eletr Enf [Internet]. 2009 [acesso em: 2017 Jun 18];11(1):158-64. Disponível em: http://www.fen.ufg.br/revista/v11/ $\mathrm{n} 1 / \mathrm{v} 1 \mathrm{ln} 1 \mathrm{a} 20 . \mathrm{htm}$

18. Castro LC, Castilho V. The cost of waste of consumable materials in a surgical center. Rev Latino-Am Enfermagem. 2013;21(6):1228-34. DOI:10.1590/0104-1169.2920.2358

19. Gil RF, Camelo SH, Laus AM. Atividades do enfermeiro de Centro de Material e Esterilização em instituições hospitalares. Texto Contextoenferm. 2013;22(4):927-34. DOI: 10.1590/S0104-07072013000400008 